K. Watanabe

Nagoya Math. J.

Vol. 83 (1981), 203-211

\title{
SOME REMARKS CONCERNING DEMAZURE'S CONSTRUCTION OF NORMAL GRADED RINGS
}

\author{
KEIICHI WATANABE
}

\section{Introduction}

In [1], Demazure showed a new way of constructing normal graded rings using the concept of "rational coefficient Weil divisors" of normal projective varieties and he showed, among other things, the following

TheOREm ([1], 3.5). If $R=\oplus_{n \geqq 0} R_{n}$ is a normal graded ring of finite type over a field $k$ and if $T$ is a homogeneous element of degree 1 in the quotient field of $R$, then there exists unique divisor $D \in \operatorname{Div}(X, Q)(X=$ $\operatorname{Proj}(R))$, such that $R_{n}=H^{0}\left(X, \mathcal{O}_{X}(n D)\right) \cdot T^{n}$ for every $n \geqq 0$. (See (1.1) for the definition of $\operatorname{Div}(X, Q)$ and $\mathcal{O}_{X}(n D)$.)

Let us denote the ring $R$ above by $R=R(X, D)$. In this note we want to consider the following problems concerning $R=R(X, D)$.

(1) What is the depth of $R$ ? In particular, when is $R$ a Macaulay ring or a Gorenstein ring?

(2) When is $R$ a rational singularity?

The paper is divided into three sections. In $\S 1$, we calculate the divisor class group of $R$. Although the contents of this section are included implicitly in [1], we need to state the results explicitly to define the canonical class $\mathrm{cl}\left(K_{R}\right)$ of $R$ in $\S 2$.

In $\S 2$, we seek the condition for $R$ to be a Macaulay ring or a Gorenstein ring. First, we express the local cohomology groups of $R$ by the cohomology groups of $\mathcal{O}_{X}(n D)(n \in Z)$. Then, using Grothendieck duality, we calculate the canonical class $\operatorname{cl}\left(K_{R}\right)$ of $R$ and, in particular, we can find the condition for $R$ to be a Gorenstein ring.

In $\S 3$, we establish a criterion for $R$ to be a rational singularity when $X$ is smooth and $\operatorname{Supp}\left(D-{ }_{[} D_{\rfloor}\right)$has only normal crossings as its singularity. (See (1.1) for the definition of $\left[D_{\rfloor}\right.$.) This criterion gives us very

Received November 28, 1979. 
abundant examples of rational singularities and that will be of some interest as there are not many examples of rational singularities known to us, yet.

\section{§1. The divisor class group of $R(X, D)$}

Throughout this paper, we shall use the following notations.

Notation (1.1). $k$ is a fixed field.

$X$ is a normal irreducible projective scheme over $k$. We assume dim $X \geqq 1$.

$k(X)$ is the rational function field of $X$.

$\operatorname{Irr}^{1}(X)$ is the set of irreducible subvarieties of codimension 1 of $X$.

$\operatorname{Div}(X)$ is the group of Weil divisors of $X$.

$\operatorname{Div}(X, Q)=\operatorname{Div}(X) \otimes_{Z} \boldsymbol{Q}$ is the group of "rational coefficient Weil divisors on $X^{\prime \prime}$. If $E=\sum r_{V} \cdot V$ and $E^{\prime}=\sum r_{V}^{\prime} \cdot V$ are elements of $\operatorname{Div}(X, Q)$, $E \geqq E^{\prime}$ means that $r_{V} \geqq r_{V}^{\prime}$ for every $V \in \operatorname{Irr}^{1}(X)$. We write

$$
\left\lfloor E_{\rfloor}=\sup \{Z \in \operatorname{Div}(X) \mid Z \leqq E\}=\sum\left[r_{V}\right] \cdot V\right.
$$

(where $[r]$ is the largest integer not larger than $r$ for $r \in Q$ ).

$\left.\mathcal{O}_{X}(E)=\mathcal{O}_{[} E_{\mathrm{J}}\right)$ for $E \in \operatorname{Div}(X, Q)$. We consider $\mathcal{O}_{X}(E)$ as a subsheaf of the constant sheaf $k(X)$.

$D=\sum p_{V} / q_{V} \cdot V$ is a fixed element of $\operatorname{Div}(X, Q)$ (where $p_{V}, q_{V} \in Z, q_{V}>0$ and $\left(p_{V}, q_{V}\right)=1$ for every $\left.V \in \operatorname{Irr}^{1}(X)\right)$ satisfying the condition;

(A) There is a positive integer $N$ such that $N D$ is an ample Cartier divisor.

Throughout this paper, we shall use the letters $D, N, p_{V}$ and $q_{V}$ in this sense.

$$
\begin{aligned}
& R=R(X, D)=\oplus_{n \geqq 0} H^{0}\left(X, \mathcal{O}_{X}(n D)\right) \cdot T^{n} \subset k(X)[T](T \text { is an indeterminate). } \\
& \mathfrak{m}=R_{+}=\oplus_{n>0} H^{0}\left(X, \mathcal{O}_{X}(n D)\right) \cdot T^{n} . \\
& C=C(X, D)=\operatorname{Spec}_{X}\left(\oplus_{n \in Z} \mathcal{O}_{X}(n D) \cdot T^{n}\right) \text { and } C^{+}=\operatorname{Spec}_{X}\left(\oplus_{n \geq 0} \mathcal{O}_{X}(n D)\right.
\end{aligned}
$$

$\left.\cdot T^{n}\right)$. Note that $C$ is an open subscheme of $C^{+}$. We put $S^{+}=C^{+}-C$. $C$ and $C^{+}$have the natural $G_{m}$-actions induced by the gradings. We have the natural homomorphism $\Psi: C^{+} \rightarrow \operatorname{Spec}(R)$ which maps $C$ isomorphically onto $\operatorname{Spec}(R)-\{\mathfrak{m}\}$ and contracts $S^{+}$to the point $\mathfrak{m}$. Note that $\Psi$ is a projective morphism. In fact, $C^{+}$is isomorphic to $\operatorname{Proj}\left(R^{\natural}\right)$ in the notation of E.G.A. Chapter II, 8.2.

$$
\begin{aligned}
& \pi: C \rightarrow X \text { and } \pi^{+}: C^{+} \rightarrow X \text { be the canonical projections. } \\
& F_{V}=\pi^{-1}(V)_{\text {red }} \in \operatorname{Irr}^{1}(C) \text { for } V \in \operatorname{Irr}^{1}(X) .
\end{aligned}
$$


$\mathrm{Cl}(Y)$ (resp. $\mathrm{Cl}(R)$ ) is the divisor class group of a normal variety $Y$ (resp. of $R$ ).

$P(Y)$ (resp. $P(R)$ ) is the group of principal divisors of $Y$ (resp. of $R$ ).

Let us put the letter $H$ to show that something on $R$ or on $C$ is stable under the $\boldsymbol{G}_{m}$-action. For example, $H \operatorname{Div}(R)$ is the subgroup of $\operatorname{Div}(R)$ consisting of the homogeneous divisors of $R$ and $H \operatorname{Irr}^{1}(C)$ is the set of irreducible subsets of codimension 1 on $C$ which are stable under the $\boldsymbol{G}_{m}$-action on $C$.

Now, we will recall some facts to calculate $\mathrm{Cl}(R)$.

(1.2) As $C \simeq \operatorname{Spec}(R)-\{\mathfrak{m}\}$ and as $\operatorname{dim} R \geqq 2(\operatorname{dim} R=\operatorname{dim} X+1)$, $\mathrm{Cl}(R) \simeq \mathrm{Cl}(C)$. Also, we have $\mathrm{Cl}(R) \simeq H \operatorname{Div}(R) / H P(R)$ ([7], Proposition 7.1).

(1.3) ([1], 2.6 and 2.8) There is a natural bijection between $\operatorname{Irr}^{1}(X)$ and $H \operatorname{Irr}^{1}(C)$ given by $V \rightarrow F_{V}$. The mapping $\pi^{*}: \operatorname{Div}(X) \rightarrow \operatorname{Div}(C)$ is given by $\pi^{*}(V)=q_{V} \cdot F_{V}$ for $V \in \operatorname{Irr}^{1}(X)$. In particular, $\pi^{*}(D)=\sum p_{V} \cdot F_{V}$ $\in \operatorname{Div}(C)$.

By $\pi^{*}$, we can identify the group $H \operatorname{Div}(C)$ with the subgroup

$\operatorname{Div}(X, D)=\left\{\sum r_{V} \cdot V \in \operatorname{Div}(X, Q) \mid q_{V} r_{V} \in Z\right.$ for every $\left.V \in \operatorname{Irr}^{1}(X)\right\}$ of $\operatorname{Div}(X, Q)$. Note that the bijection $\operatorname{Div}(X, D) \rightarrow H \operatorname{Div}(R)$ is given by $E$ $\rightarrow \oplus_{n \in Z} H^{0}\left(X, \mathcal{O}_{X}(E+n D)\right) \cdot T^{n}$.

(1.4) If we denote the quotient field of $R$ by $Q(R), Q(R)=k(C)=$ $k(X)(T)$. Every homogeneous element of $Q(R)$ can be written in the form $f \cdot T^{n}$, where $f \in k(X)$ and $n \in Z$.

(1.5) $([1], 2.9) \operatorname{div}(T)=\pi^{*}(D)=\sum p_{V} \cdot V$ in $\operatorname{Div}(C)$.

After these observations, we can easily get the following

TheOREM (1.6). There is an exact sequence

$$
0 \longrightarrow Z \stackrel{\theta}{\longrightarrow} \mathrm{Cl}(X) \longrightarrow \mathrm{Cl}(R) \longrightarrow \operatorname{Coker}(\alpha) \longrightarrow 0
$$

where $\theta$ is given by $\theta(1)=L D\left(L=L C M\left\{q_{V} \mid V \in \operatorname{Irr}^{1}(X)\right\}\right)$ and $\alpha$ is the homomorphism $Z \rightarrow \oplus_{V} Z / q_{V} Z$ given by $\alpha(1)=\left(p_{V}\left(\bmod q_{V}\right)\right)_{V}$.

Proof. Look at the following commutative diagram 


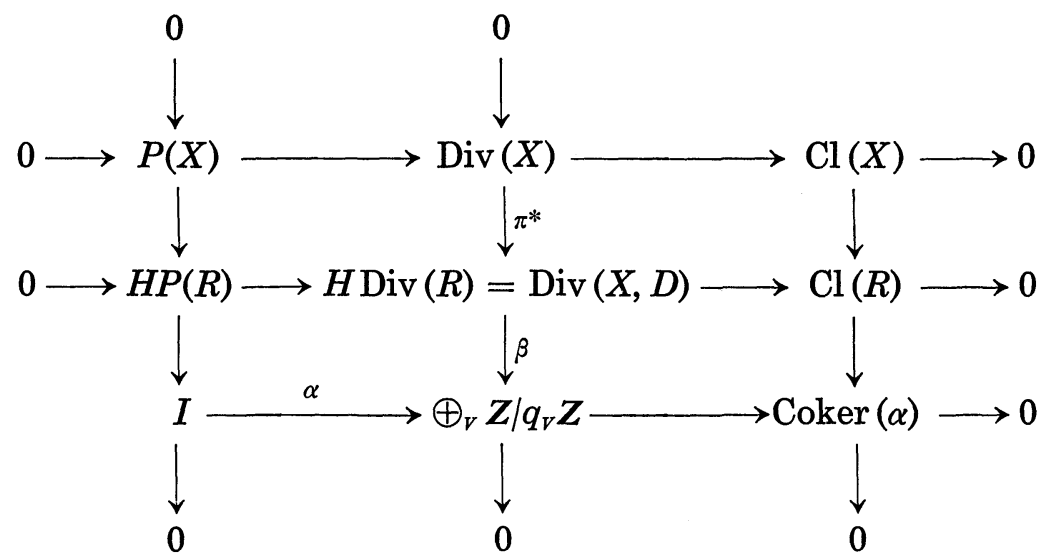

where the rows and columns are exact. The group $I \simeq Z$ is generated by $\operatorname{div}(T)$. As $\alpha(\operatorname{div}(T))=\beta\left(\pi^{*}(D)\right), \operatorname{Ker}(\alpha) \simeq Z$ and is generated by $L D$.

Corollary (1.7). $R$ is factorial if and only if $\mathrm{Cl}(X)$ is generated by $L D$ and $q_{V}$ 's are pairwise coprime.

Remark. If $k$ is algebraically closed, we can classify all factorial graded rings of dimension 2 by this method since the only normal projective curve $X$ with $\mathrm{Cl}(X) \simeq Z$ is $P^{1}$. As the result, we rediscover Theorem 5.1 of Mori [5].

\section{§2. The local cohomology groups and the canonical module of $R(X, D)$}

First, we note the following fact.

LEMMA (2.1). There is a canonical isomorphism $\widetilde{R(n)} \simeq \mathcal{O}_{X}(n D)$ on $X=$ $\operatorname{Proj}(R)$ for every $n \in Z$.

Proof. By the assumption (A), we can assume that $\mathcal{O}_{X}(m D)$ is generated by its global sections for every sufficiently large $m$. Let us take $f \in R_{m}$ and $g \in R_{m+n}$. If $m$ is a multiple of $N, m D$ is an ample Cartier divisor and $g / f$ is a section of $\mathcal{O}_{X}(n D)$ on the open set $D_{+}(f)$ of $X=\operatorname{Proj}(R)$. $\left(D_{+}(f)\right.$ is the standard notation used in E.G.A. Chapter 2.) If $m$ is not a multiple of $N$, we can take some $f^{\prime} \in R_{m^{\prime}}$ such that $m+m^{\prime}$ is a multiple of $N$. As $g / f=g f^{\prime} \mid f f^{\prime}$ on $D_{+}\left(f f^{\prime}\right)$ and as $D_{+}\left(f f^{\prime}\right)$ covers $D_{+}(f)$ when $f^{\prime}$ varies, we can reduce to the case when $m$ is a multiple of $N$. Thus we have the natural homomorphism $\widetilde{R(n)} \rightarrow \mathcal{O}_{X}(n D)$. As $\mathcal{O}_{X}(m D)$ and $\mathcal{O}_{X}((m+n) D)$ are 
generated by global sections for sufficiently large $m$, this map is surjective and the injectivity is obvious.

Proposition (2.2). There is a canonical isomorphism of graded $R$ modules

$$
H_{\mathfrak{m}}^{p}(R) \cong \bigoplus_{n \in Z} H^{p-1}\left(X, \mathcal{O}_{X}(n D)\right) \quad(p \geqq 2) .
$$

Proof. It is shown in [3], $\S 5$ that there is a canonical isomorphism

$$
H_{\mathfrak{m}}^{p}(R) \cong \underset{n \in Z}{\oplus} H^{p-1}(X, \widetilde{R(n)}) \quad(p \geqq 2) .
$$

And by (2.1), $\widetilde{R(n)} \cong \mathcal{O}_{X}(n D)$ for every $n \in Z$.

CoRollary (2.3). $\operatorname{depth} R=p+1$, where $p$ is the minimal positive integer such that $H^{p}\left(X, \mathcal{O}_{X}(n D)\right) \neq 0$ for some $n \in Z$.

CoRollaRY (2.4). $\quad R(X, D)$ is a Macaulay ring if and only if $H^{p}\left(X, \mathcal{O}_{X}(n D)\right)$ $=0$ for $1 \leqq p<\operatorname{dim} X$ and for every $n \in Z$.

ExAmple (2.5). In the following cases, $R(X, D)$ is a Macaulay ring for every $D \in \operatorname{Div}(X, Q)$ satisfying the condition (A).

(a) $X$ is a curve.

(b) $X$ is a projective space or a Grassmann variety.

(c) $X$ is a smooth complete intersection in a projective space and $\operatorname{dim} X \geqq 3$.

ExAMPle (2.6). If $X$ is a rational ruled surface and if $D$ is an ample divisor on $X$, it is known that $R(X, D)$ is a Macaulay ring. But for $D \in$ $\operatorname{Div}(X, Q)$ satisfying the condition $(\mathrm{A})$, this is no longer true. For example, if $X=\boldsymbol{P}^{1} \times \boldsymbol{P}^{1}$ and $D=1 / 2 . \Delta-1 / 5 . F_{1}-1 / 5 . F_{2}$, where $\Delta$ is the diagonal and $F_{1}$ and $F_{2}$ are fibres of the first projection, then $H^{1}\left(X, \mathcal{O}_{X}(D)\right)$ $=H^{1}\left(X, \mathcal{O}_{X}\left(-F_{1}-F_{2}\right)\right) \neq 0$, while $10 . D=5 \Delta-2 F_{1}-2 F_{2}$ is an ample Cartier divisor.

Now we will calculate the canonical class $\operatorname{cl}\left(K_{R}\right)$ of $R$. Recall that $K_{R}$ is defined by $K_{R}=\left(H_{\mathrm{m}}^{d}(R)\right)^{*}$, where $d=\operatorname{dim} R=\operatorname{dim} X+1$. See [2], (2.1.2) and (1.2) for the definition of the functor ( $)^{*}$ and $K_{R}$.

First, we recall the following fact.

LEMma (2.7). If $Y$ is a normal irreducible projective variety over $k$ and if $E \in \operatorname{Div}(Y)$, then we have the nonsingular pairing 


$$
H^{n}\left(Y, \mathcal{O}_{Y}(E)\right) \times H^{0}\left(Y, \mathcal{O}_{Y}\left(K_{Y}-E\right)\right) \rightarrow k
$$

where $d=\operatorname{dim} Y$ and $\mathcal{O}_{Y}\left(K_{Y}\right)=\omega_{Y}$ is the dualizing sheaf on $Y$.

Proof. By Grothendieck duality (cf. [8], Chapter I, (1.3)), $H^{d}\left(Y, \mathcal{O}_{Y}(E)\right)$ is dual to $\operatorname{Ext}_{\mathscr{O}_{Y}}^{0}\left(\mathcal{O}_{Y}(E), \mathcal{O}_{Y}\left(K_{Y}\right)\right)=H^{0}\left(Y, \mathscr{H}_{o m_{\mathcal{O}_{Y}}}\left(\mathcal{O}_{Y}(E), \mathcal{O}_{Y}\left(K_{Y}\right)\right)\right.$ and it is easy to see that $\mathscr{H}_{\text {om }_{O_{Y}}}\left(\mathcal{O}_{Y}(E), \mathcal{O}_{Y}\left(K_{Y}\right)\right) \cong \mathcal{O}_{Y}\left(K_{Y}-E\right)$.

Theorem (2.8). The canonical module $K_{R}$ of $R$ is given by

$$
K_{R}=\bigoplus_{n \in Z} H^{0}\left(X, \mathcal{O}_{X}\left(K_{X}+D^{\prime}+n D\right)\right),
$$

where $K_{X}$ is the canonical divisor of $X$ and $D^{\prime}=\sum_{V}\left(q_{V}-1\right) / q_{V}$.

Proof. We have $\left(K_{R}\right)_{n}=\left(H_{\mathrm{m}}^{d}(R)\right)_{n}^{*}=\left(H^{d-1}\left(X, \mathcal{O}_{X}(-n D)\right)\right)^{*} \cong H^{0}\left(X, \mathcal{O}_{X}\left(K_{X}\right.\right.$ $\left.-\left[-n D_{\mathrm{J}}\right)\right)=H^{0}\left(X, \mathcal{O}_{X}\left(K_{X}+D^{\prime}+n D\right)\right)$ by (2.2) and (2.7) (where ( )* means the dual vector space. Note that $-[-n D\rfloor$ is not equal to $[n D\rfloor$ but to $\left[n D+D_{\jmath}^{\prime}\right)$. As these isomorphisms of all degrees are compatible with the multiplication of homogeneous elements of $R$, we get the desired result.

CoRollary (2.9). If $R$ is a Macaulay ring, $R$ is a Gorenstein ring if and only if there is an integer $a=a(R)$ such that $K_{X}+D^{\prime}-a D=\operatorname{div}(f)$ for some $f \in k(X)$.

Proof. As $R$ is a Macaulay normal domain, $R$ is a Gorenstein ring if and only if $\operatorname{cl}\left(K_{R}\right)=0$ in $\mathrm{Cl}(R)$. In the notation of (1.3) and (1.6), $K_{X}+D^{\prime} \in \operatorname{Div}(X, D)$ and $\operatorname{cl}\left(K_{R}\right)=\operatorname{cl}\left(K_{X}+D^{\prime}\right)$ by (2.8). So, $\operatorname{cl}\left(K_{R}\right)=0$ if and only if $K_{X}+D^{\prime} \in \operatorname{Div}(X, D)$ is in the image of $H P(R)$. But if $f T^{a}$ is a homogeneous element of $Q(R)(f \in k(X))$, the image of $\operatorname{div}\left(f T^{a}\right)$ in $\operatorname{Div}(X, D)$ is $\operatorname{div}_{X}(f)+a D$ by (1.5).

Remark (2.10). In [2], (3.1.4), we have defined the invariant $a(R)$ of $R$ by

$$
a(R)=-\min \left\{m \mid\left(K_{R}\right)_{m} \neq 0\right\}=\max \left\{m \mid\left(H_{\mathrm{m}}^{d}(R)\right)_{m} \neq 0\right\}
$$

and showed that if $R$ is a Gorenstein ring, then $K_{R}=R(a(R))$. It is easy to show that the integer $a(R)$ in (2.9) coincides with this definition.

Remark (2.11). If $R$ is a Macaulay ring, then $X$ is a Macaulay scheme. In fact, if $R$ is a Macaulay ring, so is the $N$-th Veronese subring $R^{(N)}=$ $R(X, N D)$. As $N D$ is an ample Cartier divisor, we can say that $X$ is a Macaulay scheme by [2], (5.1.10). But even if $R$ is a Gorenstein ring, $X$ 
need not be a Gorenstein scheme. For example, if $R=k[U, V, W]$, where we put $\operatorname{deg}(U)=\operatorname{deg}(V)=1$ and $\operatorname{deg}(W)=n$, then $X=\operatorname{Proj}(R)$ is a Gorenstein scheme if and only if $n=1$ or 2 .

\section{§3. A criterion for rational singularities}

In this section, we assume $\operatorname{char}(k)=0$ since the definition of rational singularities is not founded yet in positive characteristics. Recall that $R$ is a rational singularity if for some (or, equivalently, every) resolution $\Phi: Y \rightarrow \operatorname{Spec}(R)$, the higher direct images $R^{p} \Phi_{*}\left(\mathcal{O}_{Y}\right)=0$ for all $p>0$. We will say that a scheme has only rational singularities if every singularity of it is a rational singularity.

The key lemma of this section is the following.

LEMMA (3.1). If $X$ and $D$ satisfy the following conditions, then the scheme $C^{+}=C^{+}(X, D)$ has only rational singularities.

(1) $X$ has only rational singularities.

(2) If $x \in X$ is a singular point, then $D$ is a Cartier divisor on some neighborhood of $x$.

(3) If $x \in X$ is a smooth point, then there is a regular parameter system $\left(z, \cdots, z_{d}\right)$ of the local ring $\mathcal{O}_{X, x}$ such that $\left.\operatorname{Supp}\left(D-{ }_{[} D\right]\right)$ is defined by $z_{1} \cdots z_{t}(t \leqq d)$ at $x$.

Proof. First, notice that if $E$ is a Cartier divisor on $X, C^{+}(X, D)$ has only rational singularities if and only if so does $C^{+}(X, D+E)$ as the problem is local with respect to $X$. If $\left.\left.\mathcal{O}_{X}\right|_{U} \cong \mathcal{O}_{X}(D)\right|_{U}$ for some open set $U$ of $X$, then $\left.C^{+}(X, D)\right|_{U} \cong U \times A^{1}$. Thus $C^{+}(X, D)$ has only rational singularities over some neighborhood of a singular point $x$ of $X$ by (1) and (2). If $x$ is a smooth point of $X$, we may assume that $D=\sum_{i=1}^{t} p_{i} / q_{i} \cdot V_{i}$ near $x$, where $\left(p_{i}, q_{i}\right)=1,0<p_{i}<q_{i}, 0 \leqq t \leqq d$ and the defining equation of $V_{i}$ is $z_{i}$ at $x$ by (3). Then,

$$
\begin{aligned}
C^{+}(X, D) \times_{X} \operatorname{Spec}\left(\mathcal{O}_{X, x}\right)=\operatorname{Spec}\left(\mathcal { O } _ { X , x } \left[z_{1}^{-a_{1}} \cdots z_{t}^{-a_{t}} \cdot T^{n} \mid n p_{i} \geqq a_{i} q_{i}\right.\right. \\
\text { for } i=1, \cdots, t]) .
\end{aligned}
$$

As the completion of this ring is isomorphic to that of a normal semigroup ring, this ring is known to be a rational singularity ([4], Chapter $I, \S 3$ ).

Remark (3.2). If $\left.\operatorname{Supp}\left(D-{ }_{[} D\right]\right)$ has "bad" singularities, $C^{+}$does have non-rational singularities even if $X$ is smooth. For example, let $U=$ $A^{2}(u, v)$ and $D=1 / q \cdot Z$, where $Z$ is defined by $f(u, v)=0$. Then $C^{+}=$ 
Spec $\left(k\left[u, v, T, f^{-1} T^{q}\right]\right)$ is a hypersurface in $A^{4}(u, v, T, w)$ defined by $T^{q}-$ $w \cdot f(u, v)=0$, which is not a rational singularity if $q$ and $\operatorname{deg}(f)$ are large.

TheOREm (3.3). If $X$ and $D$ satisfy the conditions of (3.1) and if $\Phi: Y$ $\rightarrow \operatorname{Spec}(R)$ is a resolution of $\operatorname{Spec}(R)$, then we have canonical isomorphisms

$$
R^{p} \Phi_{*}\left(\mathcal{O}_{Y}\right) \cong \underset{n \geq 0}{\oplus} H^{p}\left(X, \mathcal{O}_{X}(n D)\right)
$$

for all $p \geqq 0$. In particular, $R$ is a rational singularity if and only if $R$ is a Macaulay ring and the invariant $a(R)$ is negative.

Proof. As $R^{p} \Phi_{*}\left(\mathcal{O}_{Y}\right)$ does not depend on the choice of the resolution $\Phi$, we may assume that $\Phi$ is the composition of $\Theta: Y \rightarrow C^{+}$and $\Psi: C^{+} \rightarrow$ $\operatorname{Spec}(R)$. As $C^{+}$has only rational singularities, $R^{p} \Theta_{*}\left(\mathcal{O}_{Y}\right)=0(p>0)$ and we have isomorphisms

$$
R^{p} \Phi_{*}\left(\mathcal{O}_{Y}\right) \cong R^{p} \Psi_{*}\left(\mathcal{O}_{+}\right) \cong H^{p}\left(C^{+}, \mathcal{O}_{C+}\right) \cong H^{p}\left(X, \underset{n \geq 0}{\oplus} \mathcal{O}_{X}(n D)\right) \cong \underset{n \geqq 0}{\oplus} H^{p}\left(X, \mathcal{O}_{X}(n D)\right)
$$

by the definition of $C^{+}$.

Example (3.4). If $X$ is a curve, the conditions of (3.1) are always satisfied. So, $R$ is a rational singularity if and only if $a(R)<0$. This has been proved by Pinkham [6].

Example (3.5). Let $X=\boldsymbol{P}^{d}$ and $H_{i}(i=1, \cdots, s)$ be hyperplanes of $X$ in general position. If $D$ is a rational coefficient linear combination of $H_{i}$ 's and satisfies the condition (A), $X$ and $D$ satisfy the conditions of (3.1) and $R(X, D)$ is a rational singularity if and only if $\operatorname{deg}\left({ }_{\lfloor} n D_{\rfloor}\right) \geqq-d$ for every $n \geqq 0$. For example, let $p, q$ be positive integers with $(p, q)=1$, $H, H_{1}, \cdots, H_{p}$ be hyperplanes in general position and $D=b H+\sum_{i=1}^{p} a / q \cdot H_{i}$, where $a, b$ are integers which satisfy $a p+b q=1$. Then $R=R(X, D) \cong$ $k\left[S, T_{0}, \cdots, T_{d}\right] /\left(S^{q}-h_{1} \cdots h_{p}\right)$, where $T_{0}, \cdots, T_{d}$ are homogeneous coordinates of $\boldsymbol{P}^{d}$ and $h_{i}=h_{i}\left(T_{0}, \cdots, T_{d}\right)$ is the equation of $H_{i}(i=1, \cdots, p)$. As $\operatorname{deg}(S)=p$ and $\operatorname{deg}\left(T_{i}\right)=q(i=0, \cdots, d), a(R)=p q-p-(d+1) q$. So, $R$ is a rational singularity if and only if $p q-p-(d+1) q<0$.

EXAmple (3.6). Let $\left(X_{1}, D_{1}\right)$ and $\left(X_{2}, D_{2}\right)$ be pairs of a variety and a divisor satisfying the conditions of (3.1). Then, if we put $(X, D)=\left(X_{1} \times\right.$ $X_{2}, p_{1}^{*}\left(D_{1}\right)+p_{2}^{*}\left(D_{2}\right)$ ) (where $p_{i}: X \rightarrow X_{i}(i=1,2)$ are the projections), then it is easy to see that $C^{+}(X, D)$ has only rational singularities and the resulting ring $R(X, D)$ is the Segre product $R\left(X_{1}, D_{1}\right) \# R\left(X_{2}, D_{2}\right)$. As the local 
cohomology groups of Segre products are computed in [2], (4.1.5), we can easily check if $R(X, D)$ is a rational singularity or not. For example, though $R=k[x, y, z] /\left(x^{2}+y^{3}+z^{7}\right)(\operatorname{deg}(x)=21, \operatorname{deg}(y)=14$ and $\operatorname{deg}(z)$ $=6$ ) is not a rational singularity, the Segre product $R \# R^{\prime}$ is a rational singularity for any rational double point $R^{\prime}$.

ConJeCruRe (3.7). If $R$ is a Macaulay graded ring with isolated singularity and if $a(R)<0$, then is $R$ a rational singularity?

\section{REFERENCES}

[1] M. Demazure, Anneaux gradues normaux, in Seminaire Demazure-Giraud-Teissier, Singularites des surfaces, Ecole Polytechnique, 1979.

[ 2 ] S. Goto and K. Watanabe, On graded rings, I. J. Math. Soc. Japan, 30 (1978), 179-213.

[ 3 ] M. Hochster and J. L. Roberts, Rings of invariants of reductive groups acting on regular rings are Cohen-Macaulay, Adv. in Math., 13 (1974), 115-175.

[4] G. Kempf, F. Knudsen, D. Mumford and B. Saint-Donat, Toroidal embeddings, I. Lecture Notes in Math. 339, Springer, 1973.

[ 5 ] S. Mori, Graded factorial domains, Japan. J. Math., 3 (1977), 223-238.

[6] H. Pinkham, Normal surface singularities with $C^{*}$-action, Math. Ann., 227 (1977), 183-193.

[7] P. Samuel, Lectures on Unique Factorization Domains, Tata Inst. Fund. Res., Bombay, 1964.

[ 8 ] A. Altman and S. Kleiman, Introduction to Grothendieck Duality Theory, Lecture Notes in Math., 146, Springer, 1970.

Department of Mathematics

Tokyo Metropolitan University

Fukazawa, Setagaya-ku

Tokyo, 158 Japan

Current Address:

Department of Mathematics

Nagoya Institute of Technology

Gokiso-Cho, Showa-Ku

Nagoya, 466 Japan 